\title{
Face Recognition of Database of Compressed Images using Local Binary Patterns
}

\author{
Padmaja Vijay Kumar \\ Jawaharlal Nehru Technological \\ University, JNTU-AP
}

\author{
M.N.Giri Prasad,Ph.D \\ HOD and Professor-EC \\ JNTU-Anantapur-A.P
}

\author{
Padmaja.K.V,Ph.D \\ Professor, IT \\ R.V.C.E-Bangalore
}

\begin{abstract}
Local binary pattern algorithm is used in this work to determine the recognition rate for the images stored in a compressed form in the database. The images are of two types, namely, probe image and the database images. Data base images are the one present in databases like airport servers, government servers etc., whereas the probe image is the one which is being tested against the database to find the matching picture or record from the database. In this work, the data base images are compressed on the size of the image by several compression levels and each level is tested for the same probe image. The probe image is not compressed while comparison. The simulation results are presented for the recognition rate under different levels of compression.
\end{abstract}

Keywords - Face recognition, compression, image compression and face recognition, security.

\section{INTRODUCTION:}

One of the growing areas of computer vision is the domain of image processing and image recognition. Under image recognition, there are several branches, namely, pattern recognition, face recognition and object recognition [1-4]. The pattern recognition and the face recognition are very widely used in the applications like social networking, government social and criminal databases etc. For the successful face recognition, the database images [18] must have sufficient information so that when presented with the probe image, the recognition must be possible. Sometimes it is difficult to assess if enough information is actually present in the stored images so that the related records can be pulled from the databases. Majority of times, there is always excess information present in the database images, which leads to higher storage consumptions and the higher processing times. Hence optimum size of the images needs to be stored in the databases for good performance $[9,10]$.

For this purpose, the size of the images can be compressed to the required size and then stored in the databases. By compressing the size of the images, there will be loss of information but, it can be stored in large numbers and transmission of the images over the network is fast. The loss of information leads to failure of the algorithms in the recognition of the subjects in the images. Especially, the information pertaining to iris will be lost severely. Advances have been made in this area of research [5-9].

One of the major drawbacks in the face recognition using compressed images is the decompressed mode. The procedure for decompressing a compressed image for face recognition is computationally expensive and the face recognition systems would benefit if full decompression could somehow be eliminated. In other words, the face recognition is carried out while the images are in compressed mode and it would additionally increase computation speed and overall performance of a face recognition system.

The most popular compression techniques are JPEG [10] and their related transformations are Discrete Cosine Transform and Discrete Wavelet Transform. It is treated that common image compression standards such as JPEG and JPEG2000 [11]have the highest number of applications for actual usage in real life, since the image will always have to decompressed and presented to a human at some point.

It is required to store large number of images in a given space. For the image can be stored in the compressed form as well as in other forms like gray images etc. There various compression techniques $[6,8]$ available to achieve this objective but it affect the face recognition when the image is compressed with regular transformation techniques.

Also, some times the person's data set to be stored at a secret and secured place and only a representative image is stored in a computer which has access to people who are not in actual need of the real data. For example, a security guard at the airport need to identify only those people who are under surveillance and handover them to investigating agencies if the probe image and stored image matches. However, sometimes the security agencies do not want to store the actual pictures of the people in the computers operated by security guards. This is a challenging task because the representative image may not be useful for the actual face recognition and some time it may lead to misses.

For both these problems, the solution can be obtained with a single technique used in this research work. In order to address the problem of reducing the size of the file size by using regular transformation technique, one can use to reduce the frame size of the image. In this way, size of the image is reduced. However the image need to reconstructed back to its original size while doing face recognition. Since the quality of the image is altered when an image is reconstructed from lower frame size to higher frame size, it may affect its face recognition capability. In this work it is proven that face recognition technique will succeed in recognizing the faces even if the image are compressed to $10 \%$ of its original size. To address the issue mentioned in second point, the reconstructed images can be stored at the computers held by security agencies. These reconstructed images are not of good quality and the images are blurred to the maximum extent possible when reconstructed from $10 \%$ size back to its original size.

\section{LOCAL BINARY PATTERN (LBP):}

The local binary pattern $[16,17]$ face recognition algorithm is one of algorithms in which the local features are used in face recognition compared to the global features based algorithms like PCA, ICA and LDA. The algorithms consist of four parts. 
-The Tan and Triggs' illumination normalization algorithm [12] is employed initially. With this, the variation in the illumination can be eliminated.

-The local binary patterns are computed for each pixel. The LBPs are an equivalent textural description of the image.

-The histograms are created for the local features.

-The probe image and database images are compared for their histograms and the faces are recognized.

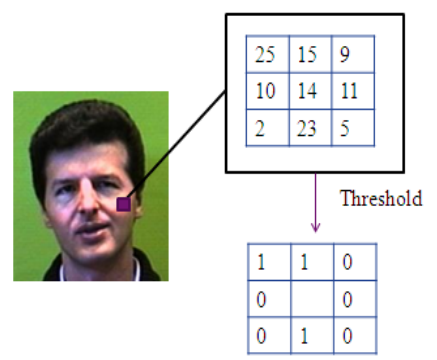

Binary code: $11000100=35$

Figure 1: LBP binary coding

\section{EXPERIMENTAL RESULTS AND DISCUSSIONS:}

Fig. 2 shows the image of 10 people whose images are considered for face recognition in this work. Two expressions of each face is taken as and stored in the database. Hence there are totally 20 images stored in the database which will be compared with the probe image to check if the probe image matches with any of the stored faces.

The stored images are considered in 10 different sizes and each size of all the 20 faces are stored in the database at a time for experimentation purpose. Fig. 3 shows the image sets of the compressed images from $90 \%$ of the original size to $10 \%$ of the original size. The left most image represents the original size, the second image is of $90 \%$ of the size, third image is $80 \%$ of the size and so on; and last one is of $10 \%$ of the size. The original size of the image is $180 \times 200$ pixels and the images shown below are only representative. When the original image is compressed to $10 \%$ of its original size, the size of the image becomes $18 \times 20$. When the probe image, which is $180 \times 200$ is presented to compare with the store image which is of size

$18 \times 20$, the dimension mis-match occurs for a the algorithm, hence the stored image is reconstructed back to the size $180 \mathrm{x}$ 200.

However, though the compressed image is reconstructed back to original size, the original image quality is not recovered as the information is lost when it is compressed and stored at $10 \%$ of the original size. In this way, a high quality probe image of size $180 \times 200$ is compared with the poor quality stored image of size $180 \times 200$. It is the interest of this work to verify to what level of compression the face recognition is successful and that size of the image can be stored in the databases instead of the original size.

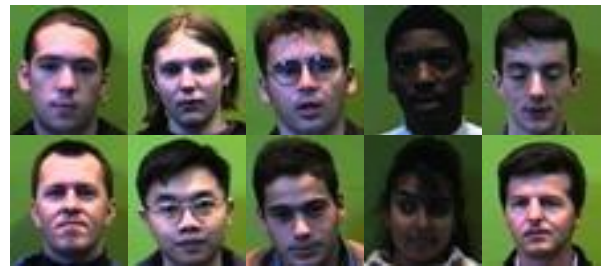

Figure 2: Images of persons considered for face recognition

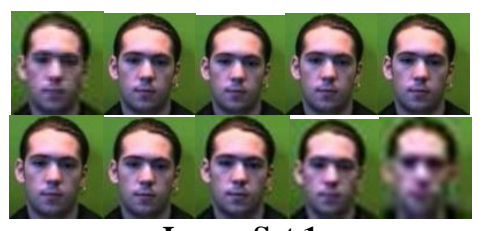

Image Set 1

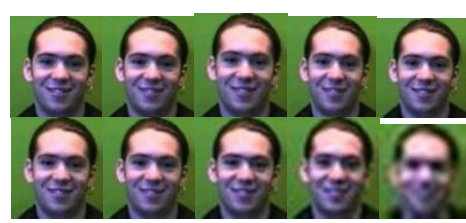

Image Set 2

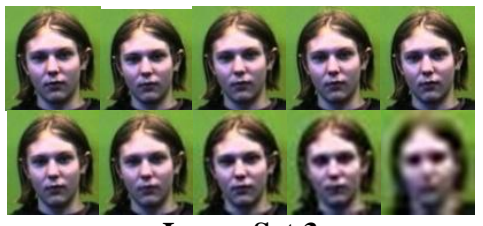

Image Set 3

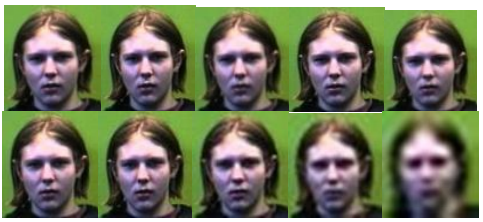

Image Set 4

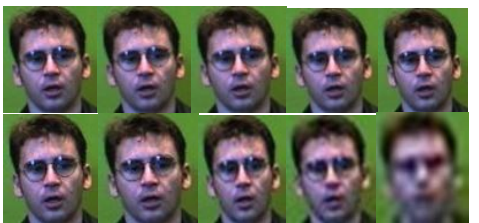

Image Set 5

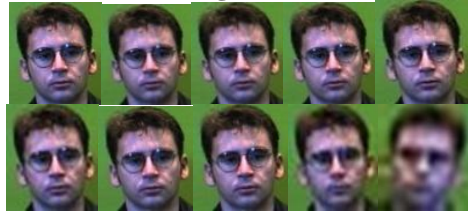

Image Set 6

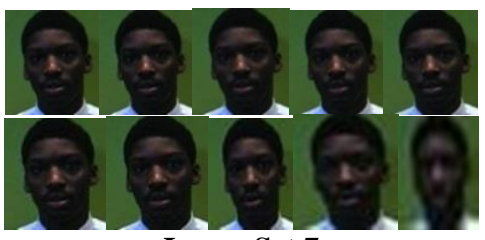

Image Set 7 


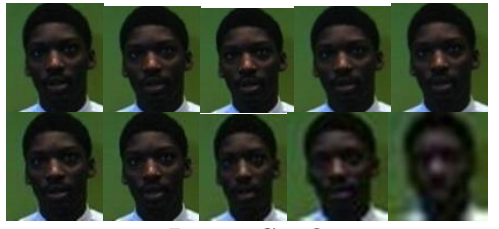

Image Set 8

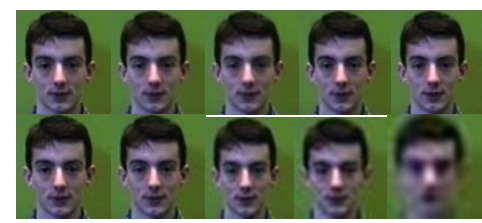

Image Set 9

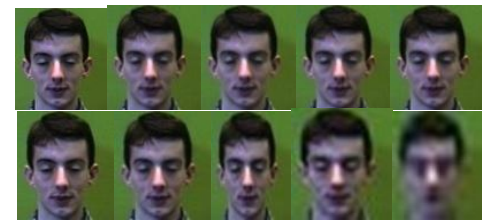

Image Set 10

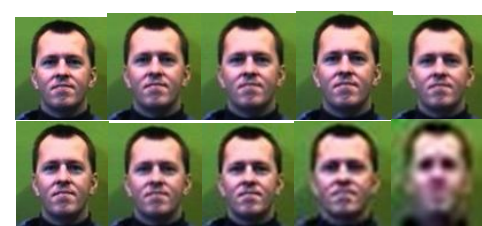

Image Set 11

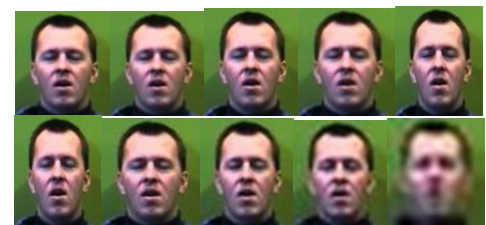

Image Set 12

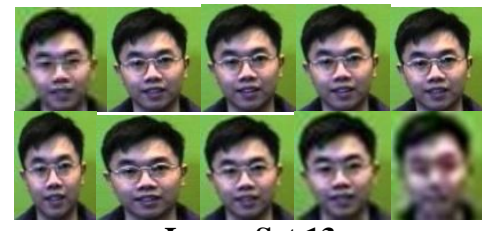

Image Set 13

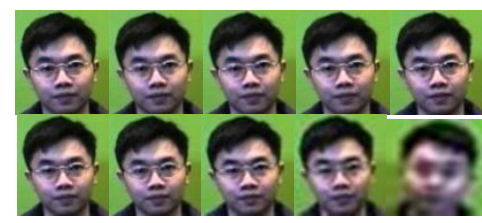

Image Set 14

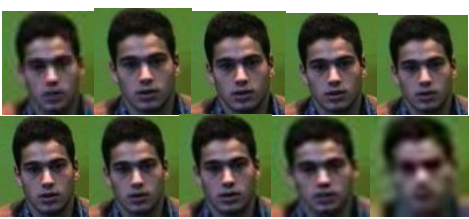

Image Set 15

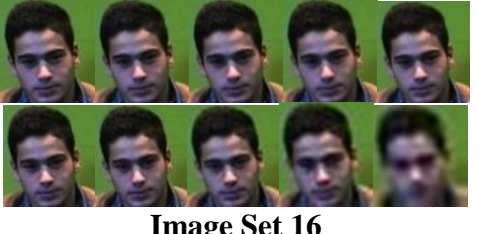

Image Set 16
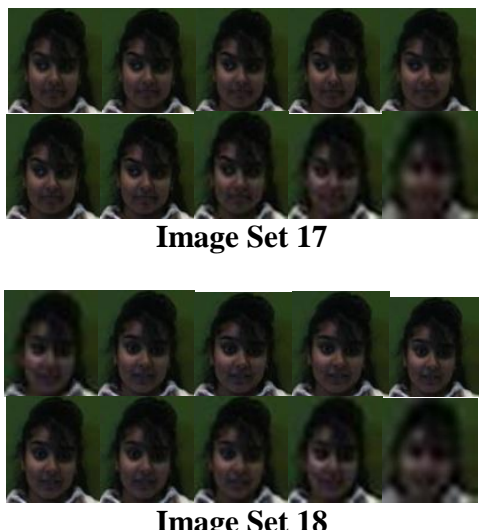

Image Set 18

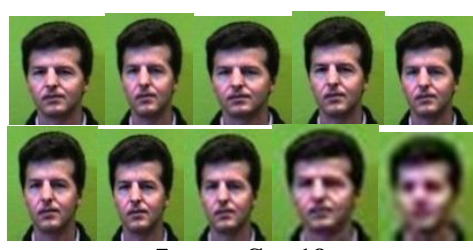

Image Set 19

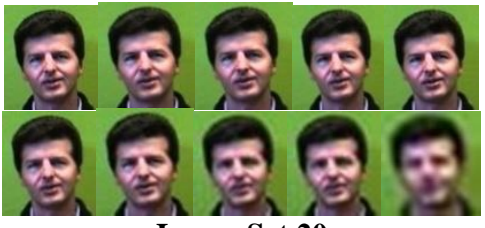

Image Set 20

Figure 3: Image sets original and compressed in size from $90 \%$ to $10 \%$ of the size from left to right.

The face recognition is successful when the probe images are presented to compare with the stored images. For all probe images, the face recognition was successful for compression sizes upto $10 \%$ of the original size, which gave the recognition rate up to $80 \%$. When the compression is carried out below $10 \%$, the face recognition was very poor. Hence the cases below 10\% compression are not presented here.

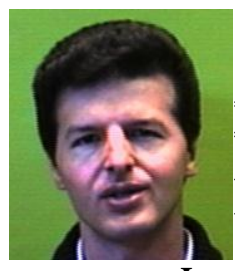

Image 1 

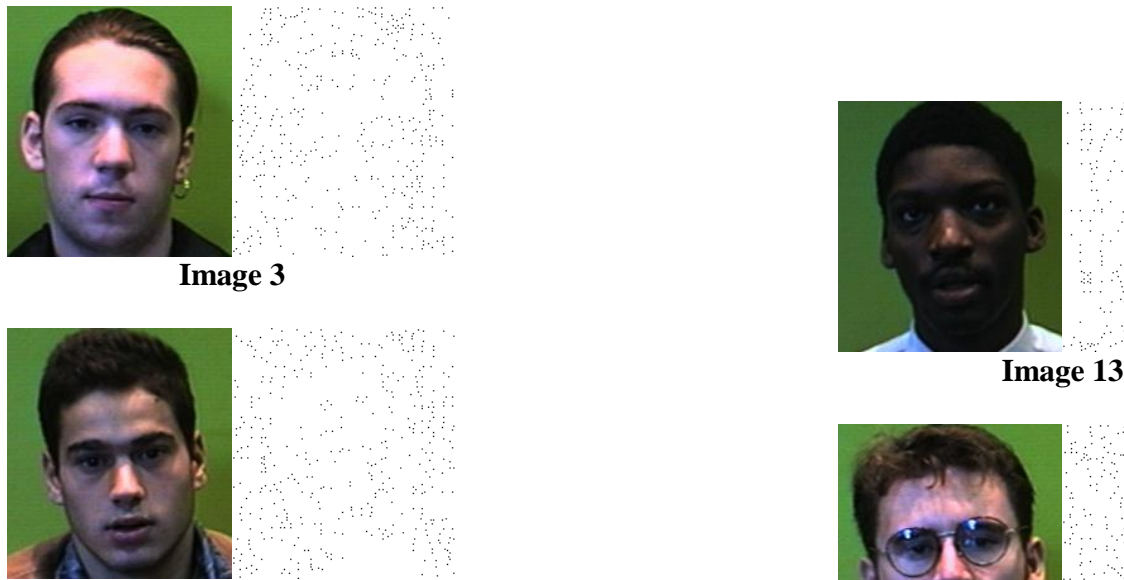

Image 5

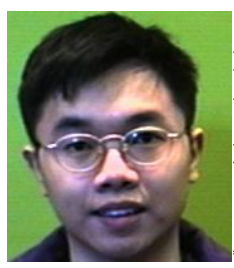

Image 7

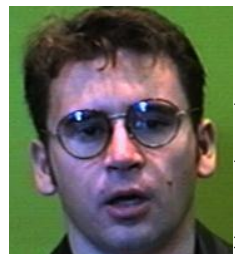

Image 15

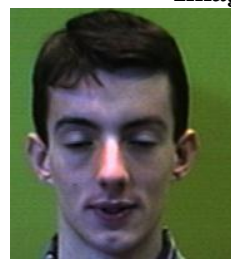

Image 17
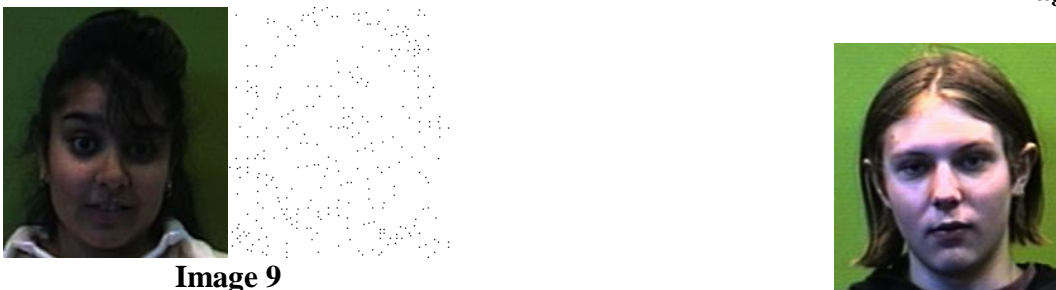

Image 19

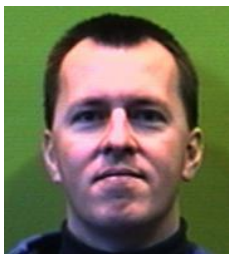

Image 11

Figure 4: Original images and transformed images after LBP on a gray image

After performing the face recognition using the LBP algorithm, it was identified that the all the images are recognized correctly when the database images are original in size, that is, the image quality of the database images and the probe images are same. 
TABLE1: Simulation Results Summary Recognition Rate;

\begin{tabular}{|c|c|c|c|}
\hline $\begin{array}{c}\text { Compressed size with } \\
\text { respect to original size }\end{array}$ & Recognition rate & $\begin{array}{c}\text { Un- } \\
\text { recognition } \\
\text { rate }\end{array}$ & $\begin{array}{c}\text { Faces that are } \\
\text { not recognized }\end{array}$ \\
\hline 100 & 100 & 0 & None \\
\hline 90 & 100 & 0 & None \\
\hline 80 & 80 & 20 & $\begin{array}{c}\text { Image } 8 \text { and } \\
\text { Image } 9\end{array}$ \\
\hline 70 & 90 & 10 & Image 9 \\
\hline 60 & 90 & 10 & Image 9 \\
\hline 50 & 90 & 10 & Image 9 \\
\hline 40 & 100 & 0 & None \\
\hline 30 & 100 & 0 & None \\
\hline 20 & 100 & 0 & None \\
\hline 10 & 80 & 20 & Image 3 and \\
Image 7 \\
\hline
\end{tabular}

When the sizes of the database images are reduced to $90 \%$ of its original size, then also, all the images are correctly recognized. The recognition rates starts to come down after compressing the size to $80 \%$ and less than $80 \%$ of the original size.
Under $80 \%$ of the original size, there are two images, namely, image 8 and image 9 are not correctly recognized, hence resulting a recognition rate of $80 \%$ and unrecognized rate of $20 \%$. For a compression size of $70 \%, 60 \%$ and $50 \%$ of the size, there is only one image which is not recognized, that is Image 9. The recognition rate for these cases, $90 \%$ and unrecognized rate is $10 \%$. For cases of $40 \%, 30 \%$ and $20 \%$, the recognition rate is $100 \%$ and all the images are correctly recognized. For $10 \%$ of the original size, again there are two misses resulting in $80 \%$ and $20 \%$ of recognition and recognition rates respectively. 


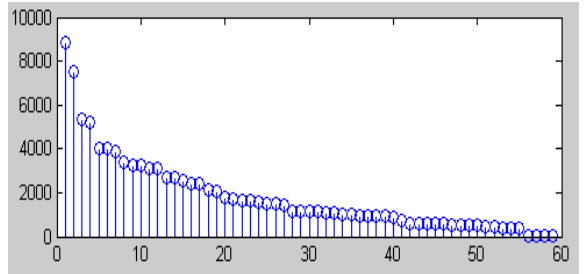

Figure 5:LBP Histogram for Image 1 for original Size

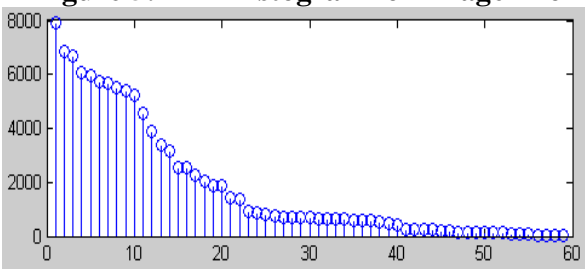

Figure 6:LBP Histogram for Image 1 for $10 \%$ of original Size

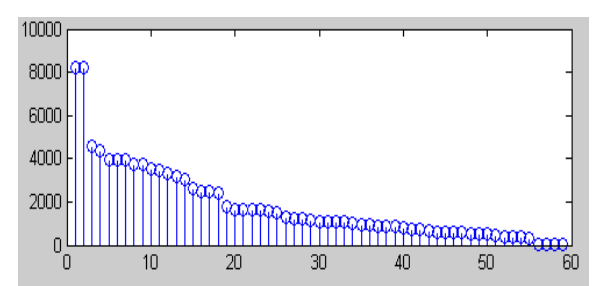

Figure 7: LBP Histogram for Image 2 for original Size

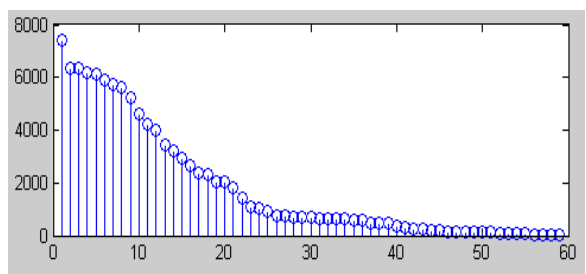

Figure 8: LBP Histogram for Image 2 for $10 \%$ of original

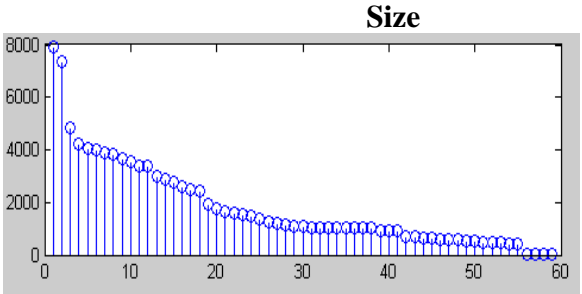

Figure 9: LBP Histogram for Image 3 for original Size

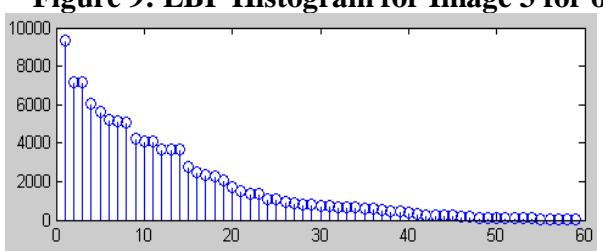

Figure 10: LBP Histogram for Image 3 for $10 \%$ of original Size

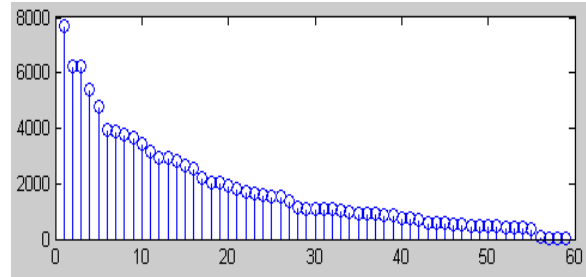

Figure 11: LBP Histogram for Image 4 for original Size

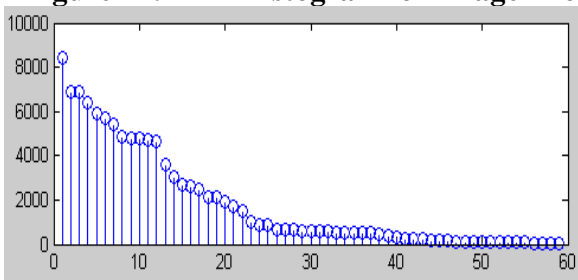

Figure 12: LBP Histogram for Image 4 for $10 \%$ of original Size

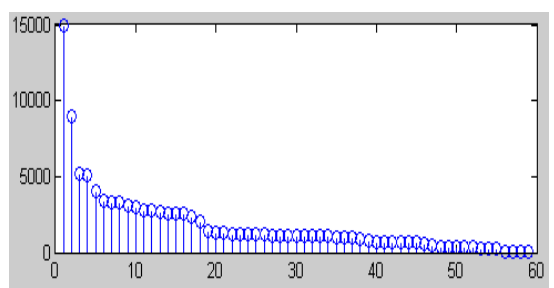

Figure 13: LBP Histogram for Image 5 for original Size

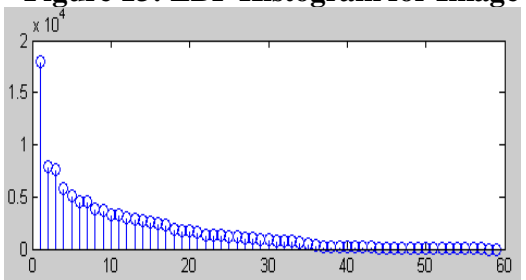

Figure 14: LBP Histogram for Image 5 for $10 \%$ of original Size

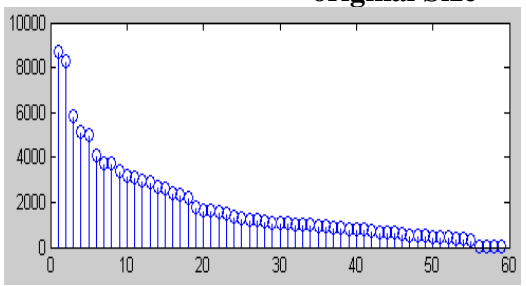

Figure 15: LBP Histogram for Image 6 for original Size

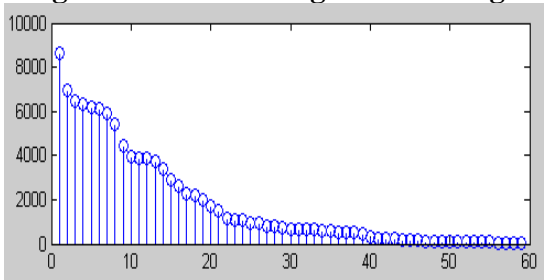

Figure 16: LBP Histogram for Image 6 for $10 \%$ of original Size 


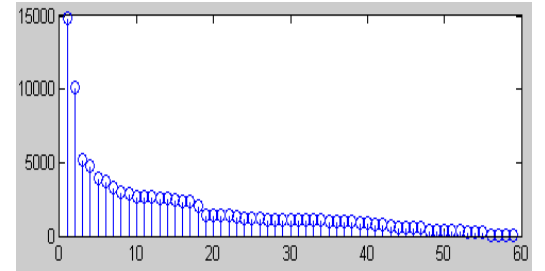

Figure 17: LBP Histogram for Image 7 for original Size

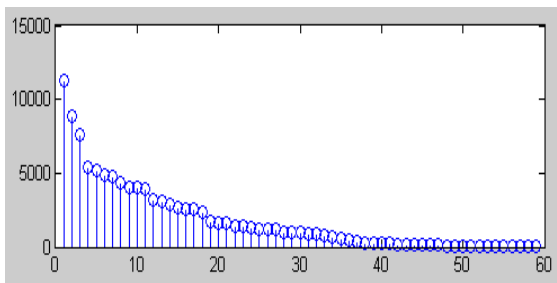

Figure 18: LBP Histogram for Image 7 for $10 \%$ of original Size

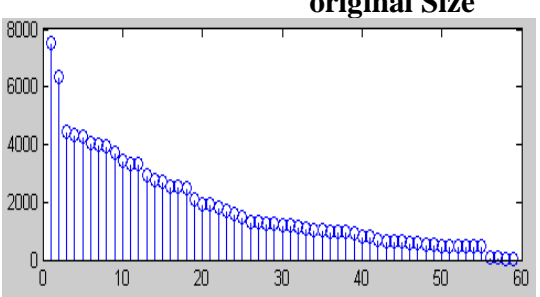

Figure 19: LBP Histogram for Image 8 for original Size

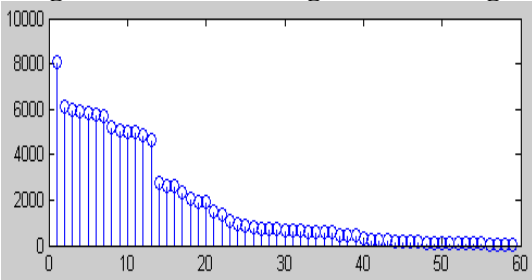

Figure 20: LBP Histogram for Image 8 for $10 \%$ of original Size

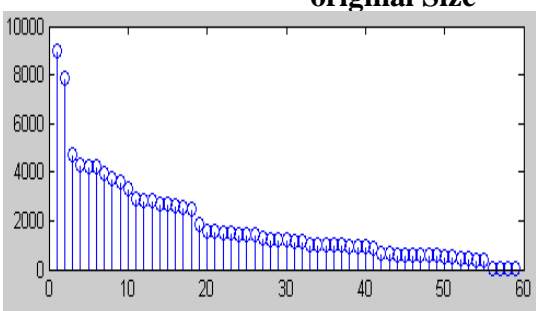

Figure 21: LBP Histogram for Image 9 for original Size

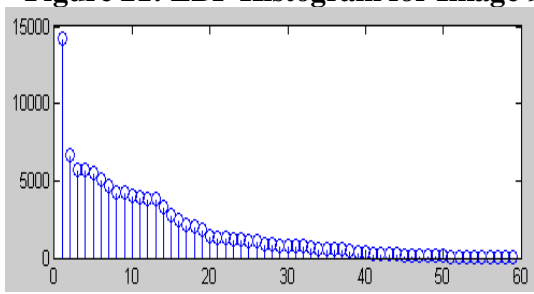

Figure 22: LBP Histogram for Image 9 for $10 \%$ of original Size

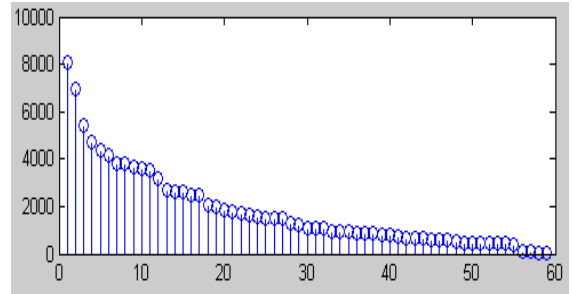

Figure 23: LBP Histogram for Image 9 for original Size

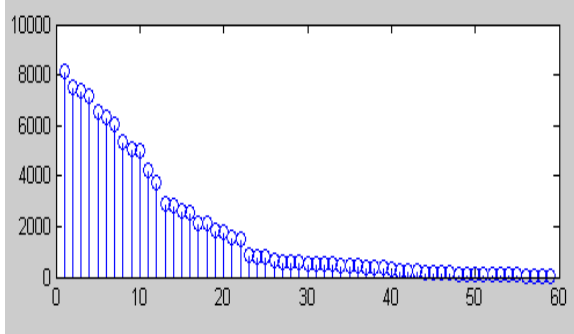

Figure 24: LBP Histogram for Image 10 for $10 \%$ of original Size

The Figs. 5 to 24 show the histograms of the images for the original size and the images of the size of $10 \%$ of the original size. The histograms are calculated and the Euclidean distance is calculated for all the database images with respect to the probe image. Whichever has the least distance, that image is considered as the equivalent image in the database. However, if the subject of the probe image does not exist in the database, then it recognizes the image with least Euclidean distance as the equivalent image. One can observe despite huge variation in the histogram which are arranged in the descending order here, the face recognition is successful. The histogram are not sorted when calculating the Euclidean distance, hence the variation between histograms should not affect the face recognition in any way. What matters here is the total variation of the histograms when computed from pixel to pixel.

\section{CONCLUSIONS:}

In this work, the performance of the local binary pattern algorithm is measured for the compressed images of the database. The images are compressed from $90 \%$ of the original size to $10 \%$ with an interval of $10 \%$. The maximum success rate is $100 \%$ for the cases like $100 \%, 90 \%, 40 \%, 30 \%$ and $20 \%$ of the compressed images. The recognition rate is $90 \%$ for compressed sizes of $70 \%, 60 \%$ and $50 \%$. It is $80 \%$ for compression sizes of $80 \%$ and $10 \%$. The recognition rate does not vary linearly with respect to the compressed size. It is random as one can notice in Table 1 . This is because the histograms are calculated based on the pixel values surrounding the reference pixel and these pixels vary with respect to the compressed size. 


\section{REFERENCES:}

[1] Zhao W., Chellappa R., Rosenfeld A., Phillips P.J., Face Recognition: A Literature Survey, ACM Computing Surveys, Vol. 35, Issue 4, December 2003, pp. 399-458

[2] Delac K., Grgic M., A Survey of Biometric Recognition Methods, Proc. of the 46th International Symposium Electronics in Marine, ELMAR-2004, Zadar, Croatia, 1618 June 2004, pp. 184-193

[3] Li S.Z., Jain A.K., ed., Handbook of Face Recognition, Springer, New York, USA, 2005

[4] Delac, K., Grgic, M. (eds.), Face Recognition, I-Tech Education and Publishing, ISBN 978-3-902613-03-5, Vienna, July 2007, 558 pages

[5] Rakshit, S., Monro, D.M., An Evaluation of Image Sampling and Compression for Human Iris Recognition, IEEE Trans. on Information Forensics and Security, Vol. 2, No. 3, 2007, pp. 605-612

[6] Matschitsch, S., Tschinder, M., Uhl, A., Comparison of Compression Algorithms' Impact on Iris Recognition Accuracy, Lecture Notes in Computer Science Advances in Biometrics,

Vol. 4642, 2007, pp. 232-241

[7] Funk, W., Arnold, M., Busch, C., Munde, A., Evaluation of Image Compression Algorithms for Fingerprint and Face Recognition Systems, Proc. from the Sixth Annual IEEE Systems, Man and Cybernetics (SMC) Information Assurance Workshop, 2005, pp. 72-78

[8] Mascher-Kampfer, A., Stoegner, H., Uhl, A., Comparison of Compression Algorithms' Impact on Fingerprint and Face Recognition Accuracy, Visual Communications and Image Processing 2007 (VCIP'07), Proc. of SPIE 6508, 2007, Vol. 6508, 650810, 12 pages

[9] Elad, M., Goldenberg, R., Kimmel, R., Low Bit-Rate Compression of Facial Images, IEEE Trans. on Image Processing, Vol. 16, No. 9, 2007, pp. 2379-2383

[10] Skodras A., Christopoulos C., Ebrahimi T., The JPEG 2000 Still Image Compression Standard, IEEE Signal Processing Magazine, Vol. 18, No. 5, September 2001, pp. $36-58$

[11] Wallace G.K., The JPEG Still Picture compression Standard, Communications of the ACM, Vol. 34, Issue 4, April 1991, pp. 30-44.

[12] X. Tan and B. Triggs, "Enhanced local texture feature sets for face recognition under difficult lighting conditions," Lecture Notes in Computer Science, vol. 4778, p. 168, 2007.

[13] Phillips, P., Grother, P., Micheals, R.J., Blackburn, D.M., Tabassi, E., Bone, J.M.:

Face recognition vendor test 2002 results. Technical report (2003)

[14] Gong, S., McKenna, S.J., Psarrou, A.: Dynamic Vision, From Images to FaceRecognition. Imperial College Press, London (2000)

[15] Phillips, P.J., Wechsler, H., Huang, J., Rauss, P.: The FERET database andevaluation procedure for face recognition algorithms. Image and Vision Computing $M$ 16 (1998) 295-306
[16] C. Shan, S. Gong, P.W. McOwan, Robust facial expression recognition usinglocal binary patterns, in: IEEE International Conference on Image Processing(ICIP), Genoa, vol. 2, 2005, pp. 370-373.

[17] S. Liao, W. Fan, C.S. Chung, D.-Y.Yeung, Facial expression recognition usingadvanced local binary patterns, tsallis entropies and global appearancefeatures, in: IEEE International Conference on Image Processing (ICIP), 2006,pp. 665-668.

[18] X. Feng, M. Pietikäinen, T. Hadid, Facial expression recognition with localbinary patterns and linear programming, Pattern Recognition and ImageAnalysis 15 (2) (2005) 546-548.

[19] G. Zhang, X. Huang, S.Z. Li, Y. Wang, X. Wu, Boosting local binary pattern (lbp)-based face recognition, in: Chinese Conference on Biometric Recognition

(SINOBIOMETRICS), 2004, pp. 179-186.

[20] G. Zhao, M. Pietikäinen, Dynamic texture recognition using local binarypatterns with an application to facial expressions, IEEE Transactions on PatternAnalysis and Machine Intelligence 29 (6) (2007) 915-928.

[21] T. Ahonen, A. Hadid, M. Pietikainen, "Face Recognition with LocalBinary Patterns", Computer Vision Proceedings, ECCV 2004, LectureNotes in Computer Science 3021, Springer, 469-481.

22] O. Lahdenoja, M. Laiho, A. Paasio, "Reducing the Feature Vector Lengthin Local Binary Pattern based Face Recognition", Proceedings of theIEEE International Conference on Image Processing (ICIP 2005), Genova,Italy.

[23] P.J. Phillips, H. Wechsler, J. Huang, P. Rauss, "The FERET Databaseand Evaluation Procedure for Face Recognition Algorithms", Image andVision Computing, 16, 1998, 295-306.

[24] T. Ahonen, A. Hadid, and M. Pietik"ainen. Face recognition with local binary patterns. In Proc.8th European Conference on Computer Vision (ECCV), Prague, Czech Republic, pages 469-481,2004

[25] X. Huang, S.Z. Li, and Y. Wang. Shape localization based on statistical method using extendedlocal binary pattern. In Proc. Third International Conference on Image and Graphics (ICIG),Hong Kong, China, pages 184-187, 2004

[26] H. Jin, Q. Liu, H. Lu, and X. Tong. Face detection using improved LBP under bayesian framework.In Proc. Third International Conference on Image and Graphics (ICIG), Hong Kong, China, pages 306-309, 2004.

[27] K. Jonsson, J. Matas, J. Kittler, and Y.P. Li. Learning support vectors for face verification and recognition. In 4th International Conference on Automatic Face and Gesture Recognition, pages 208-213, 2000.

[28] G. Zhang, X. Huang, S.Z. Li, Y. Wang, and X. Wu. Boosting local binary pattern (LBP)-based face recognition. In Proc. Advances in Biometric Person Authentication: 5th Chinese Conference on Biometric Recognition, SINOBIOMETRICS 2004Guangzhou, China, pages 179-186, 2004. 